\title{
Haptic rendering of rigid body collisions
}

\author{
Daniela Constantinescu, Septimiu E. Salcudean, and Elizabeth A. Croft \\ University of British Columbia \\ Vancouver, Canada \\ danielac@ece.ubc.ca, tims@ece.ubc.ca, ecroft@mech.ubc.ca
}

\begin{abstract}
This paper addresses the haptic rendering of rigid body collisions. A new method is proposed in which collision rendering is achieved in two steps. First, the haptic simulation uses a contact model whose stiffness is infinite during collisions and finite during sustained contact. This model is combined with a passive collision resolution scheme to compute collision impulses when new contacts arise. Second, the impulses are applied to the user's hand by a controller that coordinates forces and positions between the virtual environment and the haptic interface. Haptic rendering of rigid body collisions imparts forces that generate large hand accelerations when new contacts arise without requiring increased contact stiffness and damping. Experiments with a planar rigid virtual world validate the proposed approach.
\end{abstract}

\section{Introduction}

Haptically enabled computer aided mechanical design and training for assembly tasks require life-like haptic feedback to be provided to users while they manipulate rigid virtual objects. Realistic haptic feedback depends on stable and physically meaningful interaction forces to be computed and applied on the user's hand. In essence, it depends equally on the simulation (which computes the virtual forces) and on the haptic controller (which transmits them to the user).

Stable reflection of forces to the user's hand has been guaranteed by existing research through both control and simulation approaches. For example, the "virtual coupler" control [3], [1] addresses discretization effects and the relationship between the virtual world geometry and the impedance range of the haptic device. Similar to the virtual coupler, the "god-object" [16] and the "virtual proxy" [13] simulation methods decouple the simulation from the device control and enable stable point interaction with rigid virtual environments. More recent control approaches pro- pose to overcome the difficulties related to the variable simulation delay and the non-linearity of the virtual world dynamics using passivity [7] and prediction [2] concepts.

In contrast, the apparent rigidity of virtual environments has been mainly investigated through psycho-physical experiments [11], [9]. Those experiments have shown that, on contact, abrupt forces reflected to users increase the perceived rigidity of simulated objects. However, little control and simulation research has focused on methods for enhancing the apparent rigidity of virtual worlds. The earliest proposed use of abrupt forces applied to users upon contact, the "braking pulses" [14], utilize a control approach designed to improve contact stability. The braking pulses arise from a contact model with high initial damping. This apporach is suitable for point interaction with virtual environments. More recently, a simulation approach [5] reflects to users large forces that arise from a planar rigid body collision model. These forces satisfy Poisson's restitution hypothesis in a least squares sense and are not correlated to the change in kinetic energy of the virtual world.

This research proposes a new approach to haptic rendering of rigid body collisions utilizing both simulation and control techniques for rendering. The simulation approach employs a contact model with switching stiffness. The haptic controller coordinates both positions and forces between the virtual world and the device. In the simulation, multiple simultaneous collisions are resolved passively using Newton's restitution hypothesis. Corresponding impulsive forces are applied on the user's hand by the device controller.

In this paper, the contact model that enables collision rendering is presented in Section 2, followed by system dynamics in Section 3 and the passive collision resolution scheme in Section 4. Experiments validating the passivity of the proposed approach are presented in Section 5. Conclusions and future work are discussed in Section 6. 


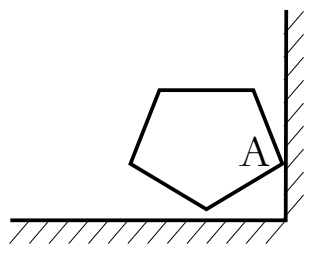

(a)

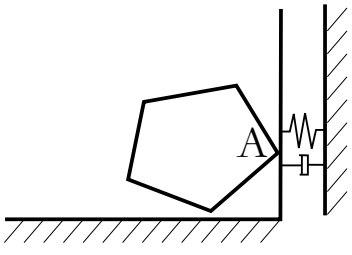

(b)

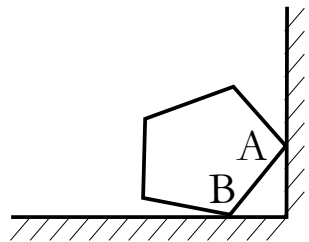

(c)

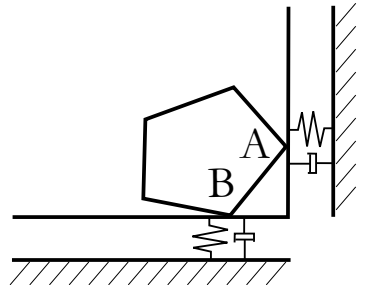

(d)

\section{Figure 1. Contacts $A$ and $B$ switch between infinite stiffness during collision and limited stiffness during sustained contact.}

\section{Contact model}

In existing physically motivated simulations, rigid body contacts are modeled as having either infinite or limited stiffness. Infinitely stiff contacts, such as those employed in constraint-based [12] and impulse-based [10] simulations, require variable step size integrators and are not suitable for haptic applications. Contacts with limited stiffness are compatible with fixed time step simulations and are used in many haptic implementations, but do not model collisions. To enable users to feel collisions, this work employs a hybrid contact whose stiffness is infinite during collisions and finite during sustained contact.

The proposed contact model is designed to allow new contacts to be emphasized perceptually (see Figure 1). When a new contact is formed, it has infinite stiffness and the virtual environment enters a collision state. After the collision has been resolved (using the methods presented in Section 4), the contact stiffness switches to a finite value and the contacting bodies may continue to move towards each other. When another contact is formed and the simulation enters a new collision state, the contact switches back to infinite stiffness unless the contacting bodies are receding from each other. This is necessary in order to ensure that the collision impulses are consistent with the rigid body constraints. In Figure 1(c), for example, the collision impulse will result in an impulsive clockwise torque (inconsistent with the constaints) unless the stiffness of contact A switches to infinity to match contact B when it occurs.

By switching to infinite stiffness upon new contact formation, the proposed contact model approximates rigid contact closer than a model with limited stiffness (i.e., a penalty-based contact model). Moreover, it enables users to feel collisions, because of the presence of high accelerations. By switching to limited stiffness during sustained contact, the model allows body interpenetration (unlike a model with infinite stiffness, i.e., a constraint-based model). This ensures its compatibility with fixed step size integrators and, therefore, with haptic applications.

\section{Virtual world dynamics}

Consider a system of $b$ rigid bodies interacting through $c$ contacts (modeled as described in Section 2), collectively called a contact group. The virtual object manipulated by the user is called body 1 or the "virtual proxy". In Cartesian coordinates, the second order dynamics of body $i$ are described by:

$$
\boldsymbol{M}_{i}\left(\begin{array}{c}
\dot{\boldsymbol{v}}_{i} \\
\dot{\boldsymbol{\omega}}_{i}
\end{array}\right)=\sum_{j=1}^{c_{i}}\left[\begin{array}{c}
\boldsymbol{I} \\
-\left(\boldsymbol{r}_{i j} \times\right)
\end{array}\right] \boldsymbol{F}_{j}+\left[\begin{array}{c}
\boldsymbol{G}_{i} \\
\mathbf{0}
\end{array}\right]-\boldsymbol{B}_{i},
$$

where bold symbols represent vectors and matrices and the following notation is used in Equation (1): $\left(\begin{array}{c}\boldsymbol{v}_{i} \\ \boldsymbol{\omega}_{i}\end{array}\right)$ and $\left(\begin{array}{c}\dot{\boldsymbol{v}}_{i} \\ \dot{\boldsymbol{\omega}}_{i}\end{array}\right)$ are the body velocity and acceleration, respectively; $\boldsymbol{I}$ is the $3 \times 3$ identity matrix; $\left(\boldsymbol{r}_{i j} \times\right)$ is the cross product operator; $\boldsymbol{r}_{i j}$ is the position of the $j$-th contact with respect to the center of mass of body $i ; \boldsymbol{F}_{j}$ is the force acting on body $i$ at the $j$-th contact; $c_{i}$ is the number of contacts of body $i$; $\boldsymbol{G}_{i}$ is the force due to gravity; $\boldsymbol{B}_{i}=\left[\begin{array}{cc}\mathbf{0} & \mathbf{0} \\ \mathbf{0} & \widehat{\boldsymbol{\omega}}_{i}\end{array}\right] \boldsymbol{M}_{i}\left(\begin{array}{c}\boldsymbol{v}_{i} \\ \boldsymbol{\omega}_{i}\end{array}\right)$ represents centripetal effects; and $\boldsymbol{M}_{i}$ is the 6-dimensional mass matrix of body $i$ (block diagonal, having the mass and the inertia tensor of body $i$ on its diagonal). Furthermore, if $\boldsymbol{J}_{i j}$ is the Jacobian of the $j$-th contact with respect to the

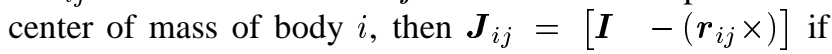
body $i$ is one of the bodies involved in the $j$-th contact, and $\boldsymbol{J}_{i j}=\mathbf{0}$ otherwise.

The second order dynamics of the contact group are obtained by considering the dynamics of the $b$ virtual objects simultaneously:

$$
\boldsymbol{M a}=\left[\begin{array}{c}
\boldsymbol{F}_{\text {user }} \\
\mathbf{0}
\end{array}\right]+\boldsymbol{G}-\boldsymbol{B}+\boldsymbol{J}_{c}^{T} \boldsymbol{F} .
$$

In Equation (2), $\boldsymbol{a}=\left(\begin{array}{lllll}\dot{\boldsymbol{v}}_{1}^{T} & \dot{\boldsymbol{\omega}}_{1}^{T} & \cdots & \dot{\boldsymbol{v}}_{b}^{T} & \dot{\boldsymbol{\omega}}_{b}^{T}\end{array}\right)^{T}$ is the acceleration of the contact group; $\boldsymbol{M}$ is a block diagonal 


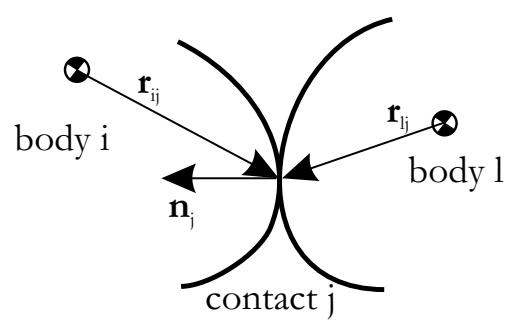

Figure 2. Contact $j$ between bodies $i$ and $l$.

matrix with $\boldsymbol{M}_{i}$ on its diagonal; $\boldsymbol{F}_{\text {user }}$ is the generalized force applied by the user at the center of mass of the virtual proxy; $\boldsymbol{G}^{T}=\left[\begin{array}{lllll}\boldsymbol{G}_{1}^{T} & \mathbf{0} & \cdots & \boldsymbol{G}_{b}^{T} & \mathbf{0}\end{array}\right]$ are forces due to gravity; $\boldsymbol{B}^{T}=\left[\begin{array}{lll}\boldsymbol{B}_{1}^{T} & \cdots & \boldsymbol{B}_{b}^{T}\end{array}\right]$ represent centripetal effects; $\boldsymbol{J}_{c}^{T}=\left[ \pm \boldsymbol{J}_{i j}^{T}\right]$ is the contact Jacobian and the sign of $\boldsymbol{J}_{i j}^{T}$ is such that the corresponding forces obey the law of action and reaction (i.e., if the $j$-th contact is between bodies $i$ and $l$ and the sign of $\boldsymbol{J}_{i j}^{T}$ is positive, then the sign of $\boldsymbol{J}_{j l}^{T}$ is negative); and $\boldsymbol{F}=\left(\begin{array}{lll}\boldsymbol{F}_{1}^{T} & \cdots & \boldsymbol{F}_{c}^{T}\end{array}\right)^{T}$ are the contact forces.

When all contacts have limited stiffness, i.e., during sustained contact, interaction forces have a component normal to the contact plane that depends on the contact stiffness, $k_{\text {contact }}$, and damping, $b_{\text {contact }}$ :

$$
\boldsymbol{F}_{n, j}= \pm\left(k_{\text {contact }} s_{j}+b_{\text {contact }} v_{r e l, j}\right) \boldsymbol{n}_{j},
$$

where $\boldsymbol{n}_{j}$ is the unit normal of the $j$-th contact; $s_{j}$ is the separation between the contacting bodies and is negative when the contact exists; and:

$$
v_{r e l, j}=\boldsymbol{n}_{j}^{T} \cdot\left(\boldsymbol{J}_{i j}\left(\begin{array}{c}
\boldsymbol{v}_{i} \\
\boldsymbol{\omega}_{i}
\end{array}\right)-\boldsymbol{J}_{l j}\left(\begin{array}{c}
\boldsymbol{v}_{l} \\
\boldsymbol{\omega}_{l}
\end{array}\right)\right)
$$

is the normal relative velocity at the contact of body $i$ with respect to body $l . v_{r e l, j}$ is positive if the objects separate (i.e., it is assumed that the contact normal points from body $l$ to body $i$ ). A component in the contact plane that models dry friction according to any friction model suitable for haptics can be added to this model as in [4].

When a new contact is formed, all non-receding contacts switch to infinite stiffness and the contact group enters a collision state. This state is resolved using the first order dynamics of the group, obtained from Equation (2) through integration:

$$
\boldsymbol{M v}=\boldsymbol{M} \boldsymbol{v}_{0}+\boldsymbol{J}_{c}^{T} \int_{t_{0}}^{t} \boldsymbol{F}_{c}=\boldsymbol{M} \boldsymbol{v}_{0},
$$

where $\boldsymbol{v}=\left(\begin{array}{lllll}\boldsymbol{v}_{1}^{T} & \boldsymbol{\omega}_{1}^{T} & \cdots & \boldsymbol{v}_{b}^{T} & \boldsymbol{\omega}_{b}^{T}\end{array}\right)^{T}$ is the postcollision velocity of the contact group, $\boldsymbol{v}_{0}$ is its pre-collision velocity, , $\mathcal{J}_{c}^{T}=\left[\boldsymbol{J}_{i j}^{T} \boldsymbol{n}_{j}\right]$ is the frictionless collision Jacobian, and $\boldsymbol{p}=\int_{t_{0}}^{t} \boldsymbol{F}_{c}$ is the impulse of the contact forces and is computed using the method presented in Section 4. In Equation (5), the impulses corresponding to the user, gravity, and centripetal forces are negligible, since collisions are considered instantaneous (i.e., $t \rightarrow t_{0}$ ).

A collision state is rendered to the user through a large force that changes the momentum of the virtual proxy over one step of the haptic simulation by the same amount as the collision impulse:

$$
\boldsymbol{F}_{c o l}=\frac{\boldsymbol{J}_{1}^{T} \int_{t_{0}}^{t} \boldsymbol{F}_{c}}{\Delta t}
$$

where $\boldsymbol{J}_{1}^{T}=\left[\begin{array}{lll}\boldsymbol{J}_{11}^{T} & \cdots & \boldsymbol{J}_{1 c}^{T}\end{array}\right]$ is the contact Jacobian of the virtual proxy and $\Delta t$ is the time step of the simulation. This can be viewed as an extension of the braking pulse of [14] from points to rigid bodies.

\section{Collision resolution}

A collision state is resolved in this work using an approach based on three assumptions: (i) that the colliding contacts are frictionless (this is a common assumption in rigid body dynamics necessary for tractability of the solution method); (ii) that all contacts have the same restitution properties, $e_{1}=\ldots=e_{c}=e$; and (iii), that Newton's restitution hypothesis applies, i.e., at each colliding contact, the pre- and post-collision normal relative velocities obey:

$$
v_{r e l}=-e v_{r e l_{0}}
$$

where the index 0 is used for pre-collision quantities and $e$ is the coefficient of restitution. $e \in[0,1]$ is a constant that describes the contact properties during collision. $e=1$ corresponds to a perfectly elastic collision (no energy loss) and $e=0$ corresponds to a perfectly plastic one (the colliding bodies do not receed from each other after collision).

From the assumption of frictionless collisions, it follows that the collision impulse $\int_{t_{0}}^{t} \boldsymbol{F}_{c}$ has components only along the directions normal to the colliding contacts:

$$
\int_{t_{0}}^{t} \boldsymbol{F}_{c}=\left(\begin{array}{c}
\int_{t_{0}}^{t} F_{1} \boldsymbol{n}_{1} \\
\vdots \\
\int_{t_{0}} F_{c} \boldsymbol{n}_{c}
\end{array}\right),
$$

and the first order dynamics of the contact group becomes:

$$
\boldsymbol{M} \boldsymbol{v}=\boldsymbol{M} \boldsymbol{v}_{0}+\mathcal{J}_{c}^{T} \boldsymbol{p} .
$$

In Equation (9), $\boldsymbol{p}=\left(\begin{array}{lll}\int_{t_{0}}^{t} F_{1} & \ldots & \int_{t_{0}} F_{c}\end{array}\right)^{T}$ is the vector of magnitudes of the collision impulses.

While Newtons' restitution hypothesis requires only that:

$$
\begin{gathered}
\boldsymbol{n}_{j}^{T}\left(\boldsymbol{J}_{i j}\left(\begin{array}{c}
\boldsymbol{v}_{i} \\
\boldsymbol{\omega}_{i}
\end{array}\right)-\boldsymbol{J}_{l j}\left(\begin{array}{c}
\boldsymbol{v}_{l} \\
\boldsymbol{\omega}_{l}
\end{array}\right)\right)= \\
=-e \boldsymbol{n}_{j}^{T}\left(\boldsymbol{J}_{i j}\left(\begin{array}{c}
\boldsymbol{v}_{i_{0}} \\
\boldsymbol{\omega}_{i_{0}}
\end{array}\right)-\boldsymbol{J}_{l j}\left(\begin{array}{c}
\boldsymbol{v}_{l_{0}} \\
\boldsymbol{\omega}_{l_{0}}
\end{array}\right)\right),
\end{gathered}
$$


for each contact $j$ and bodies $i$ and $l$, the restitution condition imposed in this work requires that:

$$
\boldsymbol{n}_{j}^{T} \boldsymbol{J}_{i j}\left(\begin{array}{c}
\boldsymbol{v}_{i} \\
\boldsymbol{\omega}_{i}
\end{array}\right)=-e \boldsymbol{n}_{j}^{T} \boldsymbol{J}_{i j}\left(\begin{array}{c}
\boldsymbol{v}_{i_{0}} \\
\boldsymbol{\omega}_{i_{0}}
\end{array}\right)
$$

for each contact $j$ and each rigid body $i$ velocity. When all $c$ colliding contacts are considered simultaneously, the restitution hypothesis becomes:

$$
\mathcal{J}_{c} \boldsymbol{v}=-e \mathcal{J}_{c} \boldsymbol{v}_{0}
$$

The momentum-impulse Equation (9) together with the restitution hypothesis in Equation (12) enable the passive resolution of the $c$ simultaneous collisions.

Two cases must be considered for resolving the collision state: (i) the case of non-redundant colliding contacts, when the frictionless collision Jacobian is full row-rank; and (ii), the case of redundant colliding contacts, when the frictionless collision Jacobian is rank-deficient. If the colliding contacts are non-redundant, the collision state is resolved by computing the collision impulse from Equation (9) after substitution from Equation (12):

$$
\boldsymbol{p}=-(1+e)\left(\mathcal{J}_{c} \boldsymbol{M} \mathcal{J}_{c}^{T}\right)^{-1} \mathcal{J}_{c} \boldsymbol{v}_{0} .
$$

Simultaneous redundant collisions are resolved similarly, by using $\left(\mathcal{J}_{c} \boldsymbol{M} \mathcal{J}_{c}^{T}\right)^{\dagger}$, the pseudo-inverse of the $\mathcal{J}_{c} \boldsymbol{M} \mathcal{J}_{c}^{T}$ matrix:

$$
\boldsymbol{p}=-(1+e)\left(\mathcal{J}_{c} \boldsymbol{M} \mathcal{J}_{c}^{T}\right)^{\dagger} \mathcal{J}_{c} \boldsymbol{v}_{0}
$$

The passivity of the proposed collision resolution method (both for non-redundant and for redundant collisions) is shown in the Appendix and is validated through experiments in the next section.

\section{Experimental validation}

\subsection{System implementation}

In addition to a method for computing collision impulses, haptic rendering of rigid body collisions requires these impulses to be applied on the user's hand. Therefore, coordination of forces is necessary between the virtual world and the device. To observe this constraint, the present work proposes to use a controller that coordinates both forces and positions between the virtual proxy and the user's hand.

The validation of the passivity of the haptic collision rendering approach is performed in this section through experiments carried out using a planar haptic interaction system.
In this system, the user applies forces and torques at the center of mass of the virtual proxy through the handle of a twin pantograph planar haptic device that allows unlimited rotation [4]. The experimental virtual world consists of a rectangular virtual proxy moving in an enclosure of walls, as shown in Figure 3. A controller optimized for transparency coordinates both forces and positions between the virtual proxy and the user's hand [15].

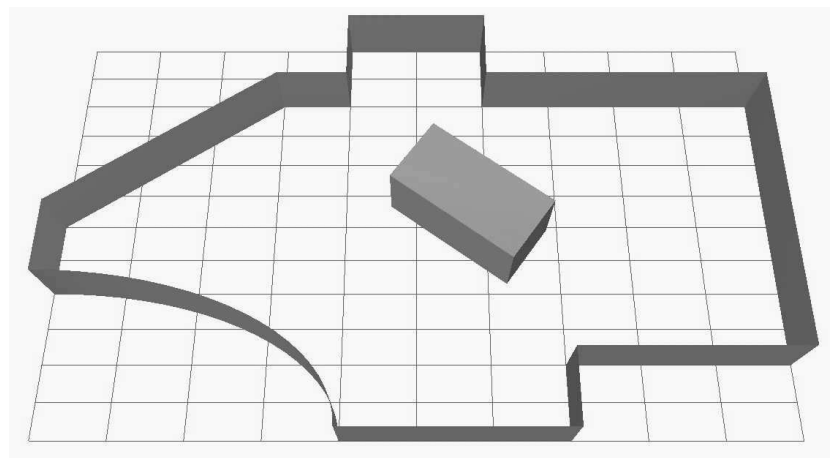

Figure 3. Experimental planar virtual world.

\subsection{Results}

The experiment consists of a peg-in-hole task: the user inserts the rectangular virtual proxy in the hole at the top of the virtual world depicted in Figure 3 (whose width is equal to the proxy's length), pushes it against the top wall several times, then extracts it, inserts and pushes it against the top again, and lets go of it. Collisions are considered perfectly plastic $(e=0)$ when they switch to infinite stiffness, and they have stiffness $k_{\text {contact }}=10000[\mathrm{~N} / \mathrm{m}]$ and damping $b_{\text {contact }}=100[\mathrm{Ns} / \mathrm{m}]$ during sustained contact. The change in proxy's kinetic energy during collisions and the number of simultaneous collisions are monitored in Figure 4 . This change is computed by subtracting the proxy's kinetic energy immediately before the collision impulses are applied to the user's hand from its kinetic energy immediately afterwards. The forces felt by the user during the experiment are shown in Figure 5.

The results in Figure 4 confirm the passivity of the haptic rendering of rigid body collisions using the approach proposed in this paper. Regardless of whether collisions are non-redundant or redundant (i.e., when four or five collisions are resolved simultaneously), the kinetic energy of the virtual proxy always decreases during a collision state if the collision impulses are computed according to Equations (13) and (14). Further experimental results validating the passivity of perfectly elastic collisions are presented in [6], while the realism of the simulation during the interaction between multiple moving bodies is the subject of current investigation. The perceptual advantage of collision 


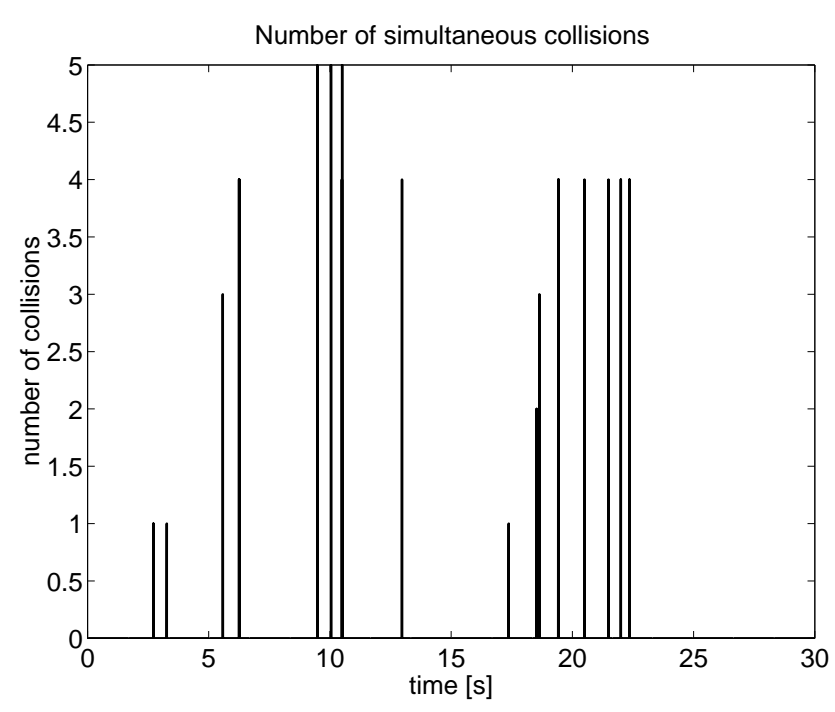

(a)

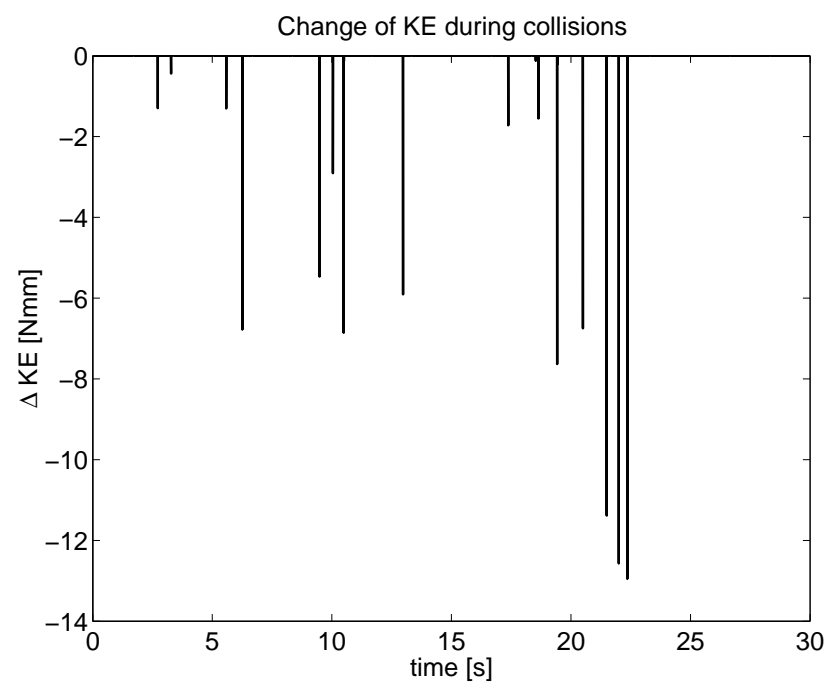

(b)

Figure 4. Change of virtual proxy's kinetic energy (KE) due to collisions during peg-in-hole task.

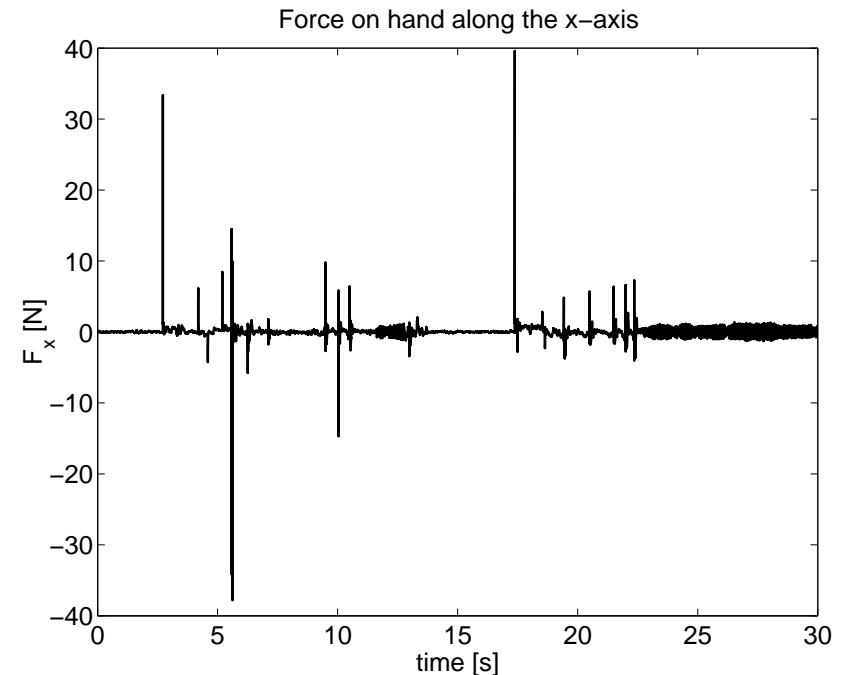

(a)

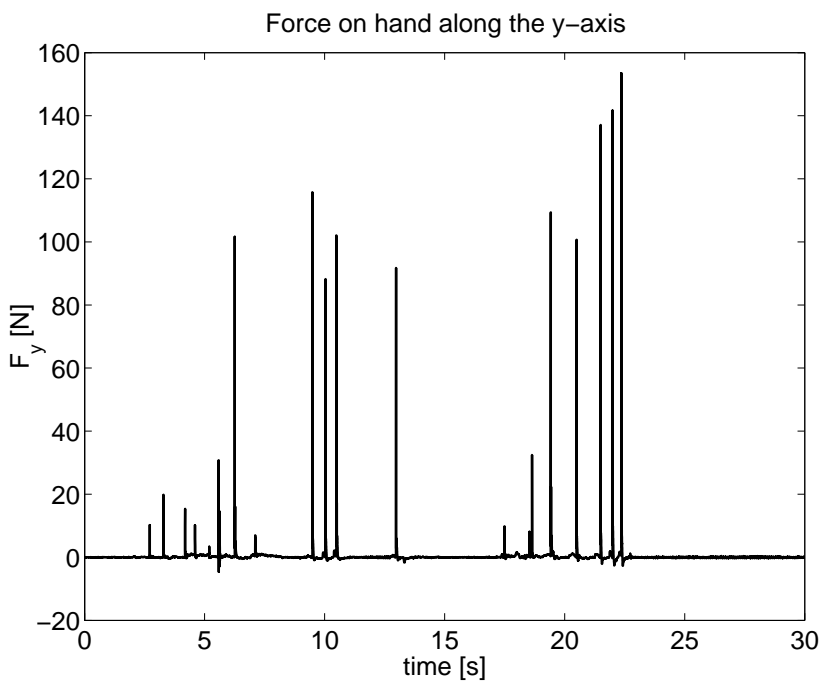

(b)

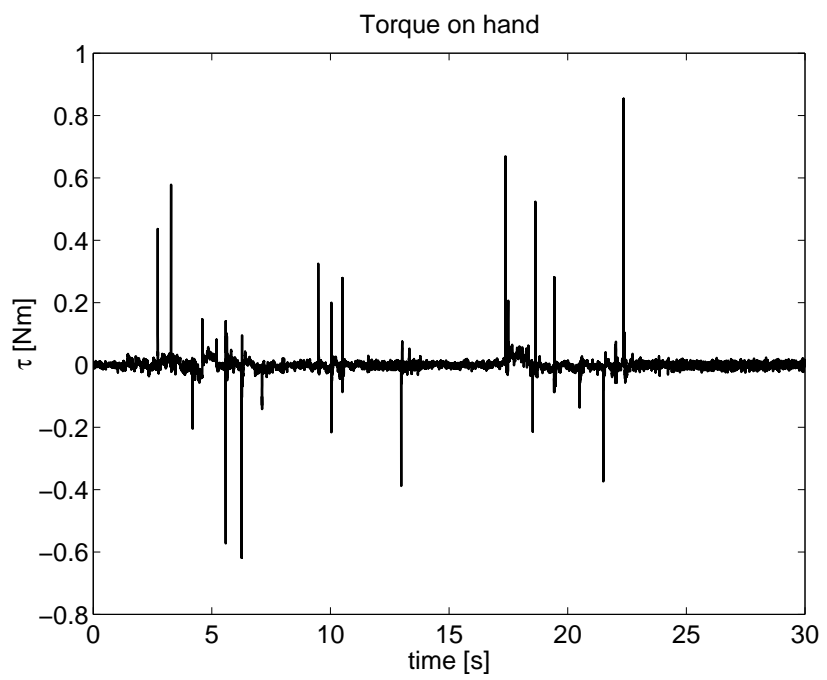

(c)

Figure 5. Forces and torques applied to the user's hand during peg-in-hole task. 
rendering is easily observed in Figure 5. As shown in this figure, the impulsive forces are an order of magnitude larger than the interaction forces developed during sustained contact. Hence, they increase the acceleration of the user's hand correspondingly and, therefore, create an improved perception of contact rigidity [9].

\section{Conclusions}

This work has proposed the haptic rendering of rigid body collisions. Users perceive collisions as large reaction forces that oppose their intended motion when new contacts arise. These large forces, computed using a new passive collision resolution technique, generate large hand accelerations without requiring increased contact stiffness and damping. The passivity of the proposed collision rendering approach is validated through haptic interaction within a planar virtual environment.

Work under way includes passive haptic rendering of rigid body collisions using local models of interaction and the extension of the proposed collision resolution scheme to multiple colliding contacts with varying restitution properties.

\section{Appendix}

This section shows that the post-collision kinetic energy of a group of rigid objects interacting through contacts is at most equal to its pre-collision kinetic energy when the collision impulse is given by Equations 13 or (14).

For non-redundant collisions, the post-collision velocity of the group is:

$$
\boldsymbol{v}=\boldsymbol{v}_{0}-(1+e) \overline{\mathcal{J}}_{c} \mathcal{J}_{c} \boldsymbol{v}_{0}=\left(\boldsymbol{I}-(1+e) \overline{\mathcal{J}}_{c} \mathcal{J}_{c}\right) \boldsymbol{v}_{0}
$$

where $\overline{\mathcal{J}}_{c}=\boldsymbol{M}^{-1} \mathcal{J}_{c}^{T}\left(\mathcal{J}_{c} \boldsymbol{M}^{-1} \mathcal{J}_{c}^{T}\right)^{-1}$ is the dynamically consistent inverse of the frictionless collision Jacobian [8]. Using the definition of $\overline{\mathcal{J}}_{c}$, the symmetry of $\boldsymbol{M}$, and some algebraic manipulation, it can be shown that:

$$
\left(\boldsymbol{I}-\mathcal{J}_{c}^{T} \overline{\mathcal{J}}_{c}^{T}\right) \boldsymbol{M} \overline{\mathcal{J}}_{c} \mathcal{J}_{c}=\mathcal{J}_{c}^{T} \overline{\mathcal{J}}_{c}^{T} \boldsymbol{M}\left(\boldsymbol{I}-\overline{\mathcal{J}}_{c} \mathcal{J}_{c}\right)=\mathbf{0}
$$

Then, it follows that:

$$
\begin{gathered}
\left(\boldsymbol{I}-\mathcal{J}_{c}^{T} \overline{\mathcal{J}}_{c}^{T}\right) \boldsymbol{M} \overline{\mathcal{J}}_{c} \mathcal{J}_{c}+\mathcal{J}_{c}^{T} \overline{\mathcal{J}}_{c}^{T} \boldsymbol{M}\left(\boldsymbol{I}-\overline{\mathcal{J}}_{c} \mathcal{J}_{c}\right)=\mathbf{0} \\
\Rightarrow \boldsymbol{M} \overline{\mathcal{J}}_{c} \mathcal{J}_{c}+\mathcal{J}_{c}^{T} \overline{\mathcal{J}}_{c}^{T} \boldsymbol{M}=2 \mathcal{J}_{c}^{T} \overline{\mathcal{J}}_{c}^{T} \boldsymbol{M} \overline{\mathcal{J}}_{c} \mathcal{J}_{c} \\
\Rightarrow(1+e) \boldsymbol{v}_{0}^{T}\left(\boldsymbol{M} \overline{\mathcal{J}}_{c} \mathcal{J}_{c}+\mathcal{J}_{c}^{T} \overline{\mathcal{J}}_{c}^{T} \boldsymbol{M}\right) \boldsymbol{v}_{0}= \\
2(1+e) \boldsymbol{v}_{0}^{T} \mathcal{J}_{c}^{T} \overline{\mathcal{J}}_{c}^{T} \boldsymbol{M} \overline{\mathcal{J}}_{c} \mathcal{J}_{c} \boldsymbol{v}_{0} \\
\geq(1+e)^{2} \boldsymbol{v}_{0}^{T} \mathcal{J}_{c}^{T} \overline{\mathcal{J}}_{c}^{T} \boldsymbol{M} \overline{\mathcal{J}}_{c} \mathcal{J}_{c} \boldsymbol{v}_{0} \quad \forall e \in[0,1] \\
\Rightarrow \boldsymbol{v}_{0}^{T} \boldsymbol{M} \boldsymbol{v}_{0} \geq \boldsymbol{v}_{0}^{T} \boldsymbol{M} \boldsymbol{v}_{0}- \\
(1+e) \boldsymbol{v}_{0}^{T} \boldsymbol{M} \overline{\mathcal{J}}_{c} \mathcal{J}_{c} \boldsymbol{v}_{0}-(1+e) \boldsymbol{v}_{0}^{T} \mathcal{J}_{c}^{T} \overline{\mathcal{J}}_{c}^{T} \boldsymbol{M} \boldsymbol{v}_{0}+ \\
(1+e)^{2} \boldsymbol{v}_{0}^{T} \mathcal{J}_{c}^{T} \overline{\mathcal{J}}_{c}^{T} \boldsymbol{M} \overline{\mathcal{J}}_{c} \mathcal{J}_{c} \boldsymbol{v}_{0} \quad \forall e \in[0,1],
\end{gathered}
$$

and, by re-arranging terms:

$$
\begin{gathered}
\boldsymbol{v}_{0}^{T} \boldsymbol{M} \boldsymbol{v}_{0} \geq \\
\boldsymbol{v}_{0}^{T}\left(\boldsymbol{I}-(1+e) \overline{\mathcal{J}}_{c} \mathcal{J}_{c}\right)^{T} \boldsymbol{M}\left(\boldsymbol{I}-(1+e) \overline{\mathcal{J}}_{c} \mathcal{J}_{c}\right) \boldsymbol{v}_{0} \\
\Rightarrow \boldsymbol{v}_{0}^{T} \boldsymbol{M} \boldsymbol{v}_{0} \geq \boldsymbol{v}^{T} \boldsymbol{M} \boldsymbol{v} .
\end{gathered}
$$

Hence, for any value of the coefficient of restitution, the post-collision kinetic energy of the contact group is at most equal to its pre-collision kinetic energy. If the simultaneous collisions are perfectly elastic, i.e., $e=1$, then the kinetic energy of the group is conserved, since:

$$
\begin{gathered}
\boldsymbol{v}=\boldsymbol{v}_{0}-2 \overline{\mathcal{J}}_{c} \mathcal{J}_{c} \boldsymbol{v}_{0}= \\
=\boldsymbol{v}_{0}-2 \boldsymbol{M}^{-1} \mathcal{J}_{c}^{T}\left(\mathcal{J}_{c} \boldsymbol{M}^{-1} \mathcal{J}_{c}^{T}\right)^{-1} \mathcal{J}_{c} \boldsymbol{v}_{0}= \\
=\boldsymbol{v}_{0}-2 \boldsymbol{v}_{0}=-\boldsymbol{v}_{0} .
\end{gathered}
$$

If the simultaneous collisions are redundant, the postcollision velocity of the contact group is:

$$
\boldsymbol{v}=\boldsymbol{v}_{0}-(1+e)\left(\mathcal{J}_{c} \boldsymbol{M}^{-1} \mathcal{J}_{c}^{T}\right)^{\dagger} \mathcal{J}_{c} \boldsymbol{v}_{0}
$$

The resolution of redundant collisions can be shown to be passive following the same reasoning as outlined in Equations (16)-(18) and replacing $\left(\mathcal{J}_{c} \boldsymbol{M}^{-1} \mathcal{J}_{c}^{T}\right)^{-1}$ with $\left(\mathcal{J}_{c} \boldsymbol{M}^{-1} \mathcal{J}_{c}^{T}\right)^{\dagger}$. The proof holds because:

$$
\begin{gathered}
\left(\mathcal{J}_{c} \boldsymbol{M}^{-1} \mathcal{J}_{c}^{T}\right)^{\dagger}= \\
=\left(\mathcal{J}_{c} \boldsymbol{M}^{-1} \mathcal{J}_{c}^{T}\right)^{\dagger} \mathcal{J}_{c} \boldsymbol{M}^{-1} \mathcal{J}_{c}^{T}\left(\mathcal{J}_{c} \boldsymbol{M}^{-1} \mathcal{J}_{c}^{T}\right)^{\dagger}
\end{gathered}
$$

\section{References}

[1] R. Adams and B. Hannaford. Stable Haptic Interaction with Virtual Environments. IEEE Trans Rob Autom, 15(3):465474, June 1999. 
[2] H. Arioui, A. Kheddar, and S. Mammar. A Model-Based Controller for Interactive Delayed Haptic Feedback Virtual Environments. J Intell Rob Syst, 37:193-207, 2003.

[3] J. Colgate, M. Stanley, and J. Brown. Issues in the Haptic Display of Tool Use. In Proc IEEE/RSJ Int Conf Intell Rob Syst, pages 140-145, Pittsburgh, 1995.

[4] D. Constantinescu, I. Chau, L. Filipozzi, S. DiMaio, and S. Salcudean. Haptic Rendering of Planar Rigid-Body Motion using a Redundant Parallel Mechanism. In Proc 2000 IEEE Int Conf Rob Autom, pages 2440-2445, Berkeley, Ca, 2000.

[5] D. Constantinescu, S. Salcudean, and E. Croft. Haptic Feedback using Local Models of Interaction. In 11th International Symposium on Haptic Interfaces for Virtual Environment and Teleoperator Systems, pages 416-421, Los Angeles, Ca, 2003.

[6] D. Constantinescu, S. Salcudean, and E. Croft. Impulsive Forces for Haptic Rendering of Rigid Contacts. In Accepted for publication at the 35th International Symposium on Robotics, Paris, France, 2004.

[7] B. Hannaford and J. H. Ryu. Time-Domain Passivity of Haptic Interfaces. IEEE Trans Rob Autom, 18(1):1-10, 2002.

[8] O. Khatib. Inertial Properties in Robotic Manipulation: An Object-Level Framework. Int J Rob Res, 13(1):19-36, 1995.

[9] D. Lawrence, L. Pao, A. Dougherty, M. Salada, and Y. Pavlou. Rate-Hardness: A New Performance Metric for Haptic Interfaces. IEEE Trans Rob Autom, 16(4):357-371, 2000.

[10] B. Mirtich and J. Canny. Impulse-based Dynamic Simulation. In K. Goldberg, P. Halperin, J.-C. Latombe, and R. Wilson, editors, Workshop Alg Found Rob, pages 407418. A.K.Peters, Boston, MA., 1994.

[11] L. Rosenberg and B. Adelstein. Perceptual Decomposition of Virtual Haptic Surfaces. In Proc 1993 IEEE Symp Res Front Virt Real, pages 46-53, Los Alamitos CA, 1993.

[12] D. Ruspini and O. Khatib. Collision/Contact Models for Dynamic Simulation and Haptic Interaction. In Proc 9th Int Symp Rob Res, pages 185-195, Snowbird, Utah, 1999.

[13] D. Ruspini, K. Koralov, and O. Khatib. The Haptic Display of Complex Graphical Environments. In Proc SIGGRAPH 97, pages 345-352, Los Angeles, CA, 1997.

[14] S. Salcudean and T. Vlaar. On the Emulation of Stiff Walls and Static Friction with a Magnetically Levitated Input/Output Device. ASME J Dyn Meas Contr, 119:127-132, March 1997.

[15] M. Sirouspour, S. DiMaio, S. Salcudean, P. Abolmaesumi, and C. Jones. Haptic Interface Control - Design Issues and Experiments with a Planar Device. In Proc 2000 IEEE Int Conf Rob Autom, pages 789-794, San Francisco, Ca, 2000.

[16] C. Zilles and J. Salisbury. A Constraint-based God Object Method for Haptic Display. In ASME Haptic Interf Virt Envir Teleop Syst, pages 146-150, Chicago, IL, 1994. 